* Corresponding author

E-mailaddress:tomtrz@prz.edu.pl

Article information

Article history: AMS-Volume16-No.2-00165-12

Received 15 January 2012

Accepted 15 July 2012

\section{Application of Genetic Algorithm for Optimization of Neural Networks for Selected Tribological Test}

\author{
Tomasz Trzepieciński* , Hirpa Lemu Gelgele ${ }^{\mathrm{a}}$ \\ * Rzeszow University of Technology, Department of Materials Forming and Processing, ul. W. Pola 2, 35-959 Rzeszów, Poland \\ a University of Stavanger, Department of Mechanical and Structural Engineering and Materials Science, 4036 Stavanger, Norway
}

\section{KEYWORDS}

Friction, friction coefficient, genetic algorithm, artificial neural networks.

\begin{abstract}
In this work was presented the method of determination of the friction coefficient by using multilayer artificial neural networks on the basis of experimental database obtained from the strip drawing test. Using genetic algorithm the optimization of number of input variables of artificial neural networks has been done. As an input parameters for training artificial neural networks following parameters has been used: surface parameters of the sheet and dies, sheet material parameters and clamping force. Some results have pointed out that genetic algorithm has been successfully appled to optimization of training set.
\end{abstract}

\section{Introduction}

Friction regimes encountered during deep drawing of thin metal sheets are very complex and depend on several parameters such as the contact pressure, sliding velocity, sheet metal and tool surface roughness, kinematics of tool motion, tool and blank material, lubrication, temperature $[1,2,3]$. One of the main factors influencing on fric-tional resistance is surface topography of deformed sheet. Friction between the sheet and tools is one of the important factors affecting the quality of drawpiece, so that the clarification of the friction is essential for modeling and analysis of sheet metal form $\neg$ ing processes. The workpiece surface topography and asperity contact is an important factor controlling the mecha $\neg$ nisms of lubrication in metal forming process.

In many research works, for describing the real frictional properties of deep drawing process, the Amontons-Coulomb simple friction model (constant coefficient of friction) is used. The Amontons-Coulomb model is true when the frictional resistance does not depend on nominal area of the contact. Furthermore it is right in limited range of contact pressures because friction in significant degree depends on sliding velocity and varied contact geometry. Measuring the frictional properties of a material always requires replicating the conditions under which the friction occurs, including the material sliding against test material, the geometry of contact, the conditions of the surfaces, and the speed of relative motion of the sliding bodies.

In the deep drawing process a few regions ex-ist, i.e. the wall, bottom and flange of the cup, with different stress state, strain state, sliding speed and friction condiations. In this regard a series of tribological tests modeled friction conditions in different parts of drawpiece were elaborated. In strip drawing test [4] coefficient of friction is de- 
termined based on measurement of other fac-tors e.g. friction force and normal force.

The aim of experimental researches was the determination of coefficient of friction for differ-ent grades of sheets used in sheetmetal working. The friction tests were carried out for wide range of contact pressures between sheet-tool interface. Considering significant amount of factors influencing frictional resistance during sheet metal forming analitycal determination of relationships utilized for calculation of friction coefficient is practically impossible. For that purpose a multi-layer artificial neural networks (ANN) were uti-lized. An important factor of correct operations of ANN is suitable selection of input variables, often is used methods of experiment optimization in order to reduce number of experiments [5]. The optimization of experimental data set used in re-searches for ANN training was carried out by using genetic algorithm (GA). There has been growing interest in applying of ANN to engineering fields for solving complicated problems including control, optimization, regression and predicting. A neural network is a nonlinear dynamic computa-tional system, capable to model extremely com-plex functions. Elaborate networks based on the abductive modeling technique are able to represent sophisticated and uncertain relationship between input and output variables. The utilization of the ANN enables the behaviour of complicated system to be modeled and predicted based on known experimental data. The ANN can solve complex nonlinear problems, with the ability of using current and historical data [6]. The purpose of the present study is to examine further ability of neural networks to predict accurately the friction coefficient value.

\section{Experimental Procedure}

WFriction tests were carried by strip drawing method placed between two fixed cylindrical rolls with equal radii (Fig. 1). The test was carried out in such a way that a strip of the sheet was clamped with specified force between two cylindrical rolls of equal radii $20 \mathrm{~mm}$. The both clamping $F_{c}$ and pulling $F_{p}$ force values were constantly recorded using electric resistance strain gauge technique, 8-channel universal amplifier of HBM's QuantumX data acquisition system and computer PC. The specimens for the friction tests were made of two brass sheet metal M63 with hardening state of $r, z 4$ and z6 as well as M90 with hardening state of z4. Samples were prepared as a strip of $20 \mathrm{~mm}$ width and about $200 \mathrm{~mm}$ length, cut along transverse directions of the sheet. The rolls were made of cold working tool steel hardened to $58 \mathrm{HRC}$. The tests were performed under the following condiations: Ra parameter of surface roughness of rolls: 0.32 ; $0.63 ; 1.25$ and $2.5 \mu \mathrm{m}$ measured along generating line of rolls, clamping force: $0.4 ; 0.8 ; 1.2 ; 1.6$ as well as $2 \mathrm{kN}$. The sliding veloc $\neg$ ity was $0.002 \mathrm{~m} / \mathrm{s}$, which is relatively high compared with the industrial values.

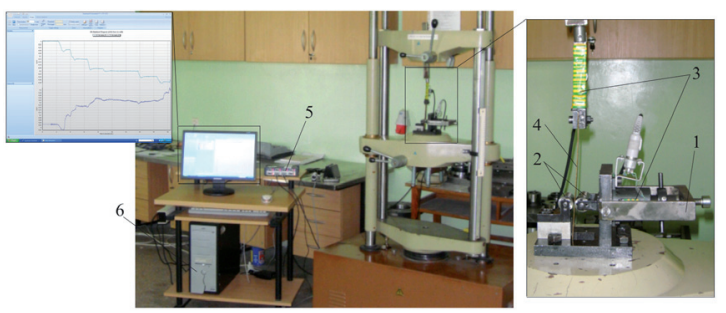

Fig. 1: View of measuring position: 1 - frame, 2 -working rolls, 3 - load cells, 4 - specimen, 5 - universal amplifier, 6-computer PC.

During the recording of the pulling and clamping forces the sheet was drawn for a distance of about $10 \mathrm{~mm}$. Next the clamping force value was increased simultaneously during tests. To realize dry conditions both rolls and sheet specimens were degreased using acetone. The mean value of the friction coefficient is determined according to (1) for the stabilized range of values of $F_{p}$ and $F_{c}$.

$\mu=\frac{F_{P}}{2 \cdot F_{C}}$

where: FP - pulling force, FC - clamping force. To determine the mechanical properties tensile test in universal testing machine was carried out. Surface roughness 3D parameters were measured by using Taylor Hobson Subtronic 3+ instrument. Table 1 presents the mechanical properties and selected spatial parameters of the sheets.

Friction tests were realized for all combinations of grade of brass, roughness of rolls and clamping forces. In this way eighty different data set for training of ANN were obtained.

\section{Artifficial Neural Networks}

The ANN's are enable to build and analyse linear and nonlinear models to solve complex regression and classification problems. Neural networks are 
used particularly to answer the purpose when dependence between inputs and outputs are very complicated. ANN consist of connected together elements named neurons which processing supplied in input information data. In general working of neural networks base on parallel processing idea. Each input signal $x_{i}$, where $i=1, \ldots, n$ is loading to neuron by weighted connections $w_{i}$ (Fig. 2). Every neuron has a threshold value specified its activation level. In kth neuron sum of input signal values $x_{n}$ multiplicationed by weight factors is calculated. Next this value is increased by external signal value which is refered as a bias term $\theta_{k}$. Calculated in this way e value is neuron acti-vation value which is converted by established activation function $\mathrm{f}_{k}(e)$ of $k$ th neuron. Value determined by activation function is output neuron value and specify nonlinear relationship between resultant input signal and output signal y neuron.

Table 1: Selected mechanical properties and surface roughness parameters of the sheets.

\begin{tabular}{|c|c|c|c|c|}
\hline \multirow[t]{2}{*}{ Parameter } & \multicolumn{4}{|c|}{ Material } \\
\hline & M63r & M63 z4 & M63 z6 & M90 z4 \\
\hline $\mathrm{C}, \mathrm{MPa}$ & 581 & 570.3 & 615 & 400 \\
\hline $\mathrm{n}$ & 0.365 & 0.143 & 0.043 & 0.040 \\
\hline $\mathrm{Sa}, \mu \mathrm{m}$ & 0.162 & 0.151 & 0.108 & 0.33 \\
\hline $\mathrm{Sq}, \mu \mathrm{m}$ & 0.205 & 0.196 & 0.137 & 0.279 \\
\hline $\mathrm{Sp}, \mu \mathrm{m}$ & 1 & 2.16 & 1.03 & 1.16 \\
\hline Ssk & 0.262 & 0.0371 & 0.191 & 0.202 \\
\hline $\mathrm{Sz}, \mu \mathrm{m}$ & 1.78 & 2.24 & 1.34 & 2.14 \\
\hline Sv, $\mu \mathrm{m}$ & 1.05 & 1.03 & 0.623 & 0.988 \\
\hline Sku, $\mu \mathrm{m}$ & 3.37 & 4.72 & 3.74 & 4.24 \\
\hline Str, mm & 0.287 & 0.153 & 0.109 & 0.175 \\
\hline SHTp, $\mu \mathrm{m}$ & 0.337 & 0.309 & 0.223 & 0.31 \\
\hline $\begin{array}{l}\mathrm{Smmr}, \\
\mathrm{mm}^{3} / \mathrm{mm}^{2}\end{array}$ & 0.00105 & 0.00103 & 0.000623 & 0.000988 \\
\hline $\begin{array}{l}S d q, \mu m / \\
\mu m\end{array}$ & 0.0201 & 0.0183 & 0.0136 & 0.0184 \\
\hline Std, ${ }^{\circ}$ & 1.5 & 1 & 1 & 1.5 \\
\hline $\mathrm{Sfd}$ & 2.5 & 2.32 & 2.4 & 2.41 \\
\hline Sdr, \% & 0.201 & 0.167 & 0.009 & 0.0169 \\
\hline Sbi & 0.316 & 0.107 & 0.172 & 0.147 \\
\hline Sal, mm & 0.074 & 0.149 & 0.088 & 0.123 \\
\hline
\end{tabular}

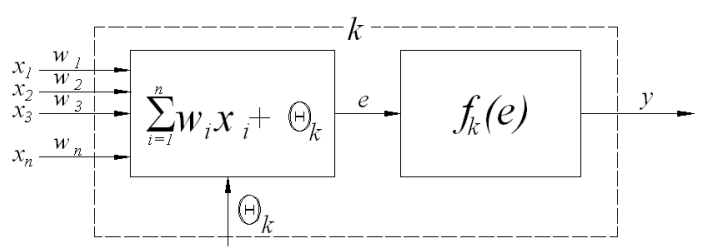

Fig. 2: Structure of nonlinear neuron $k$.

Most often applied activation functions in multilayer perceptron (MLP) is linear threshold function and its modifications. Some sort of threshold function is used: a hard limiting threshold function, a semi-linear function or a sigmoid functions. In some cases, the output of a unit can be a nonlinear function or a stochastic function of the total input of the unit.

Among nonlinear functions a hyperbolic tangent can be used with yielding output values in the range $[-1 ;+1]$. Mathematically a formula which describing an output value of neuron can be written as

$y=f\left(\sum_{i=1}^{n} w i x i\right)$

in which $f(x)=1$ when $x \geq 0, f(x)=0$ when $x<$ $0, w_{0}=\nu, u_{0}=-1, y$ - output of the neuron, $w_{i}$ - the weight of the connections which feed into neuron $j$, $x_{i}$-input signal of the neuron $i, \nu$ - the threshold of the neuron.

A single layer neural networks are characterized by the most simple structure however most often are utilized multilayer networks named multilayer perceptrons. A multilayer perceptron with a suitable number of hidden layers and neurons is theoretically sufficient to approximate any nonlinear function [7]. In order to calculate the output value of neuron of MLP Network the hyperbolic tangent function was applied:

$f(a)=\tanh (x)=\frac{e^{a}-e^{-a}}{e^{a}+e^{-a}}$

To determine weighted sum and threshold activation value of separated neurons necessary is preparation training data set consist of input signal values and corresponded values of output signals. As input signals the following input variables set was assigned:

- strength coefficient C [MPa] and strain hardening in- 
$\operatorname{dex} n$,

- roughness average parameter Ra of rolls surface [um], - surface roughness 3D parameters of the sheets, am-

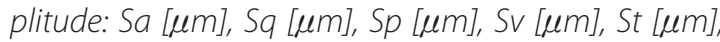
Ssk, Sku, Sz [um], superficial and volumetric: SHTp [um], Smmr $\left[\mathrm{mm}^{3} / \mathrm{mm}^{2}\right]$, Smvr $\left[\mathrm{mm}^{3} / \mathrm{mm}^{2}\right]$, spatial: Sds [number of vertex/mm2], Str, Sal [mm], Std [0], Sfd, hybrid: Sdq [um/um], Ssc [1/um], Sdr [\%], and functional: Sbi, Sci, Svi, - clamping force of rolls FC.

One of the main task necessary to build optimal model of neural network is sufficient selection of input variables which essentially influenced on the output variable value. Too large number of variables may cause information noise whereas do not taking into account even though one variable which essentially influencing output variable may lead to get wrong results. Adding in the input network the next neuron causes excessive expansion of its architecture. Simultaneously, the value of training data is increased. In turn, omission of essential variables in input can cause decreasing quality of the network. There are not universal criteria for selecting an architecture of ANN [8]. Selection of variables essentially influencing the friction coefficient value is difficult because of complex interactions of many factors particularly surface parameters which are additionally correlated each other. Application of genetic algorithms allows to select input variables without necessity to have knowledge about physical interdependences between individual input variables and output variable.

\section{Genetic Algorithms}

Genetics algorithm are based on natural selection mechanisms as well as heredity and operated on population of individuals which are potential solution of the problem. Analogous to natural conditions individuals are subjected to reproduction. Mechanisms of natural selection depend on survive of individuals the most adapted in specified environment. Only the strongest individuals survive transmitting genetic information to offspring. A data carrier about individual characteristic of individuals is chromosome, which is gene set with specified length. Most often binary representation of chromosome in computer calculation is used. The genetic encoding of a real or artificial organism is contained within their chromosomes. Each chromosome consists of a large number of genes, each uniquely located on the chromosome. Each gene in turn is composed of several alleles. The GA encoded alleles as either zero or one, represented by a single computer bit. A suitable representation of potential results should be resistant to applied genetic operators in order to after evolution can be decoded in order to find solution for input data structure. Set of many chromosomes is called population which is subjected to undergo continuous changes and depends on moment $t$ :

$$
P(t)=\left\{\nu_{1}(t), \nu_{2}(t), \ldots \nu_{n}(t)\right\}
$$

where: $n$-number of chromosomes, $\nu$ - single chromosome.

Chromosomes consist of genes deciding on hereditary one or more features. Evolution of chromosome population run by reproduction which is composed of crossover, mutation and inversion. Mechanisms of crossover and mutation of genetic information from parents lead to situation in which next generations are average better adapted to environment conditions [9]. In the crossover process genes of chromosomes of parents are joined in one or a few offspring chromosomes. Mutation lead to modification of chain by change one gene or its sequence whereas inversion reverse fragment of chromosome. The task of reproduction is to ensure of output of optimization procedure from local maxima of fitting function by variability of chromosomes. The objective of the GA is finding such solution for which the value of fitting function reaches maximum.

Algorithm worked on the initial population which equals 200 individuals with crossover coefficient $p_{k}=0.5$, mutation coefficient $p_{m}=0.1$ and with different values of coefficient of unit penalty (Table 1). The unit penalty coefficient is multiplied by a number of choosen in each mask of input variables, and next is added to the value of validation error. For optimization of number of input variables classical Holland's genetic algorithm was used. The evaluation of the population was carried out with the help of mechanism which for each solution sets in order the selection probability to new population with help of roulette selection and to new population is also loaded the best individual so far found. The task of genetic algorithm was to check the quality of network that realize the generalized regression for given set of input variables resulting from reproduction mechanism of initial population. The collection of the best sets of input data is the result of genetic 
algorithm rules (Table 2).

Increasing value of unit penalty causes the reduction of number of input variables (Fig. 3). With a large number of input variables determined by a small value of unit penalty appears a high value of a genetic algorithm error, which next starts to decrease reaching local minimum for unit penalty value equals 0.001 . Further increasing of a unit penalty value causes the increase of an error value. The high error value with a great number of variables can be explained by noise of variables which can be in certain range of value correlated with each other. Then a high value of unit penalty causes that from the quality of algorithm more important occurs the variables numbers [10]. Local increase of the error value corresponding with the value of unit penalty equals 0.0005 can be explained by the fact of appearing a variable that after removing of two variables becomes dominating correlated with other variables. For further analysis the set of 12 input variables characterized by the smallest error value was chosen.

The process of the construction of network on the base of information contained in a chromosome and following it learning of a received model must be done each time by determining of chromosome quality.

Table 2: Influence of unit penalty value on the choice of input variables by genetic algorithm.

\begin{tabular}{|c|c|c|c|c|c|c|c|c|c|c|c|c|c|c|c|c|c|c|c|c|c|c|c|c|c|c|c|}
\hline & Unit & $\mathrm{C}$ & $\mathrm{n}$ & $\tilde{\simeq}$ & $\stackrel{\pi}{\mathscr{n}}$ & $\mathscr{n}$ & की & $\vec{n}$ & $\ddot{\infty}$ & $\frac{y}{\tilde{n}}$ & $\frac{\vec{z}}{\sim}$ & $\stackrel{N}{\sim}$ & 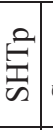 & 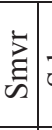 & $\underset{\mathscr{n}}{\tilde{n}}$ & $\ddot{\rightleftarrows}$ & 胥 & $\overrightarrow{\tilde{D}}$ & $\bar{\omega}$ & $\overrightarrow{\mathscr{S}}$ & $\begin{array}{l}0 \\
\tilde{n} \\
\tilde{n}\end{array}$ & $\dot{\vec{n}}$ & $\overrightarrow{\tilde{\omega}}$ & $\overline{\tilde{U}} \mid$ & $\stackrel{s}{s}$ & U & $\stackrel{\check{0}}{\stackrel{0}{0}}$ \\
\hline \multirow{6}{*}{ 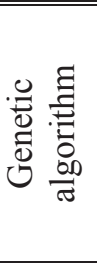 } & 0.0001 & + & + & + & + & & + & + & + & + & & + & + & + & + & + & & + & + & + & + & + & + & + & & + & 1.343 \\
\hline & 0.0002 & + & + & + & + & & + & + & + & & & + & & + & + & & & + & + & + & + & + & & & & + & 1.244 \\
\hline & 0.0005 & + & + & + & + & & + & & + & & & + & & + & + & & & + & + & & + & + & & & & + & 1.287 \\
\hline & 0.001 & + & + & + & + & & + & & + & & & & & + & & & & + & + & & + & + & & & & + & 1.232 \\
\hline & 0.002 & + & & + & + & & + & & & & & & & + & & & & + & & & + & + & & & & + & 1.326 \\
\hline & 0.004 & & & + & + & & & & & & & & & & & & & + & & & & & & & & + & 1.398 \\
\hline$I P S$ & & + & + & + & + & & + & & + & + & & + & & + & & & & +1. & + & + & +1 & + & & & & + & 1.355 \\
\hline
\end{tabular}

While the operation of the algorithm of genetic optimization the number of evaluated neuron networks is the multiplication of chromosome numbers in the population and number of considered ganaration.

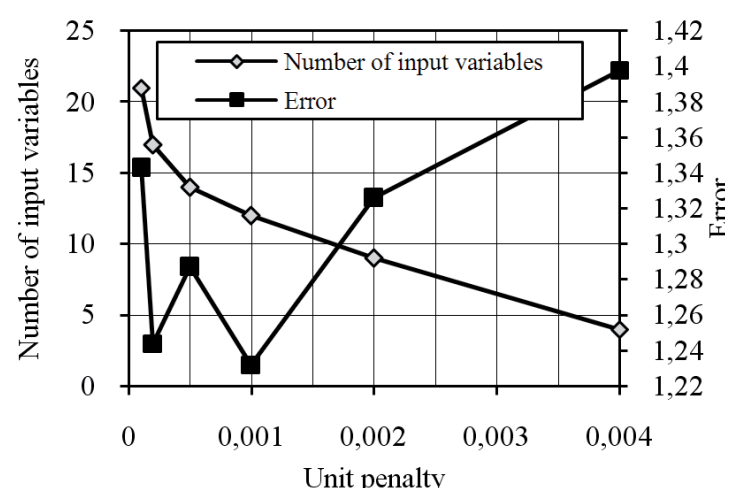

Fig. 3: Results of genetic algorithm application.

For early determined by the genetic algorithm set of input variables a series of analyses of different ANN architecture in Statistica Neural Networks were investigated. An objective of analysis was to find the network architecture ensured the small- est value of standard deviation ratio in connection with high value of Pearson's correlation coefficient $R$ [11]. Results of these analyses were compared with results of network model based on input variables determined by Intelligent Problem Solver (IPS) builtin Statistica Neural Networks (Table 2). On the basis of loading data Intelligent Problem Solver determines set of "the best" neural network architecture. In Statistica there is not particular information about mathematical nature of input variable selection by using IPS.

Among all experimental set consisted of input data and corresponded with output signal were separated $20 \%$ of data which were assigned to testing set (Ts). Data vectors from a testing set did not participate in training process and served to ANN prognostic evaluation purpose. From the remaining set of experimental data belonging to training set (Tr) was separated 10\% of data which were assigned to validation set $(V)$. Data from this group were used for independent check of back propagation (BP) training algorithm. The learning rate was equal to 0.1 [12]. To overlearning prevent the learning process braked off when value of verification round 
mean square error (5) for validation set was stopped dropping [10].

$$
R M S=\sqrt{\frac{\sum_{i=1}^{N}(z i-y i)^{2}}{N}}
$$

where: $N$ - number of vectors of training set, $y_{i}-$ signal of output signal for ith standard, $z_{i}$ - expected signal of output neuron for ith standard.

Table 3 shows the regression statistics of "the best" neural networks for input variables determined by genetic algorithm (MLP 12:12-12-1:1, MLP 12:12-141:1), Intelligent Problem Solver (MLP 15:15-10-1:1) and using entire variable set do not put to preprocessing process (MLP 25:25-12-1:1). The model with the lowest values of standard deviation ratio in connection with highest value of Pearson-R correlation is network MLP 12:12-14-1:1 which regression statistics are slight better in comparison with network MLP 12:12-12-1:1 and considerably better from MLP 15:15-10-1:1 taking into account all analysed input variables.

One of the methods to determine the im-portance of influence of particular input variables on the value of explained variable is sensivity analysis (Table 4) which also can be used for choosing input variables. The criterion of sensivity is the value of the network error after removing this variable. Bigger importance of the variable determines bigger value of the network error. Tak-ing into account the training set clamping force $\mathrm{FC}$, roughness average parameter $\mathrm{Ra}$ of rolls surface and maximum peak height Sp have the biggest influence on the coefficient of friction value.

Table 3: Influence of unit penalty value on the choice of input variables by genetic algorithm.

\begin{tabular}{||c|c|c|c|c|c|c|c|c|c|c|c|c||}
\cline { 2 - 13 } \multicolumn{1}{c|}{} & \multicolumn{2}{c|}{ MLP 12:12-12-1:1 } & \multicolumn{2}{c|}{ MLP 12:12-14-1:1 } & \multicolumn{3}{c|}{ MLP 15:15-10-1:1 } & \multicolumn{3}{c|}{ MLP 25:25-12-1:1 } \\
\hline \hline Set & $\mathrm{Tr}$ & $\mathrm{V}$ & $\mathrm{Ts}$ & $\mathrm{Tr}$ & $\mathrm{V}$ & $\mathrm{Ts}$ & $\mathrm{Tr}$ & $\mathrm{V}$ & $\mathrm{Ts}$ & $\mathrm{Tr}$ & $\mathrm{V}$ & $\mathrm{Ts}$ \\
\hline Error S. D. & 0.005 & 0.005 & 0.008 & 0.006 & 0.007 & 0.011 & 0.005 & 0.010 & 0.009 & 0.008 & 0.900 & 0.013 \\
\hline $\begin{array}{c}\text { Abs error } \\
\text { mean }\end{array}$ & 0.0009 & 0.002 & 0.0001 & 0.001 & 0.008 & 0.0009 & 0.003 & 0.004 & 0.002 & 0.009 & 0.012 & 0.005 \\
\hline RMS error & 0.005 & 0.006 & 0.009 & 0.004 & 0.005 & 0.010 & 0.007 & 0.009 & 0.012 & 0.008 & 0.013 & 0.011 \\
\hline S. D. ratio & 0.149 & 0.253 & 0.250 & 0.153 & 0.248 & 0.170 & 0.196 & 0.298 & 0.171 & 0.275 & 0.351 & 0.285 \\
\hline $\begin{array}{c}\text { Correlation } \\
\text { R }\end{array}$ & 0.989 & 0.968 & 0.973 & 0.997 & 0.976 & 0.996 & 0.980 & 0.992 & 0.993 & 0.923 & 0.918 & 0.937 \\
\hline \hline
\end{tabular}

Table 4: Sensivity analysis for MLP 12:12-14-1:1.

\begin{tabular}{|c|c|c|c|c|}
\hline \multirow[t]{2}{*}{ Variable } & \multicolumn{2}{|c|}{ Training set } & \multicolumn{2}{|c|}{ Validation set } \\
\hline & Error & Ratio & Error & Ratio \\
\hline C & 0.0161 & 1.720 & 0.0156 & 1.940 \\
\hline$n$ & 0.0184 & 1.996 & 0.0180 & 2.243 \\
\hline Ra & 0.0275 & 2.911 & 0.0234 & 2.910 \\
\hline Sa & 0.0116 & 1.227 & 0.0149 & 1.851 \\
\hline Sp & 0.0252 & 2.710 & 0.0305 & 3.793 \\
\hline St & 0.0168 & 1.784 & 0.0129 & 1.612 \\
\hline Smvr & 0.0152 & 1.665 & 0.0124 & 1.545 \\
\hline Std & 0.0241 & 2.585 & 0.0201 & 2.498 \\
\hline Sfd & 0.0114 & 1.225 & 0.0159 & 1.986 \\
\hline SsC & 0.0172 & 1.880 & 0.0176 & 2.197 \\
\hline Sdr & 0.0151 & 1.724 & 0.0120 & 1.498 \\
\hline FC & 0.0340 & 3.616 & 0.0360 & 4.477 \\
\hline
\end{tabular}

Analyzing the networks with genetic selection of input variables it can be noticed that increase of neurons number in the hidden layer causes the increase of error value on the output of network for each data set.

Less number of neurons in the hidden layer should influence of larger network ability to elim-inate the noises coming from input data greater ability of generalization. Ability of generalization is the quality of generalization of acquired knowledge in the learning process to cases from behind the learning set.

Results of this research shows excellent agreement between experimental data and out-comes of neuron models for all range of input variable used for the training. (Fig. 4). This will allow to reduce or eliminate expensive and time-consuming carrying out of experimental tests in order to determine friction coefficient value. The application of the ANN also allows to eliminate search of complicated dependence between pa-rameters influencing on the 
friction and the fric-tion coefficient value.

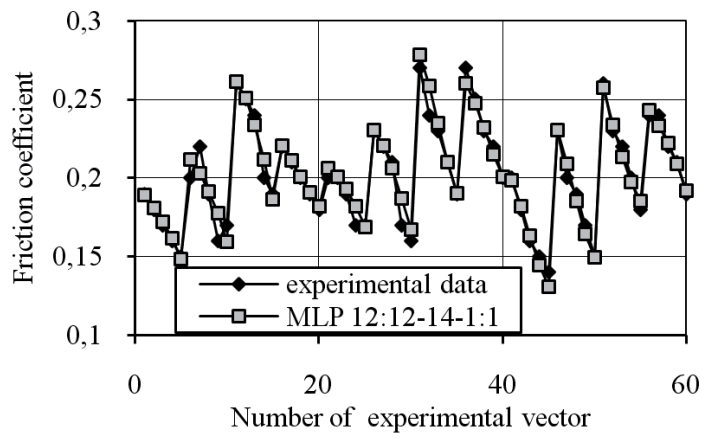

Fig. 4: Comparison of friction coefficient value determined experimentally and using MLP 12:12-14-1:1.

\section{Conclusion}

IUsing of optimization methods of input variables numbers allows to avoid the time-consuming testing of neuron models with different architecture in order to find the optimum network for specific task. Regression statistics of neuron model based on the genetic selection of input variables and using Intelligent Problem Solver are considerably better than models without preprocessing of input data. The most effective in working are neural networks with not extended structure. Proper selection of input variables consisting in rejection of variables which are correlated with each other, influencing in small degree on the explained variable or even rejection of variables that have important information influence on the reduction of network structure. Analyzed network models characterized by unexpectedly good results taking into account that number of input signals and responding to them output signal were limited to eighty.

\section{Acknowledge}

The project was realized with financial support provided by Island, Liechtenstein and Norway and was co-financed by European Economic Area and Norwegian Financial Mechanism under the Scholarship and Training Fund.

\section{References}

[1] Darendeiler H., Akkok M., Yucesoy C.A., Effect of variable coefficient on sheet metal drawing, Tribol. Int., vol. 35, no. 2, 2002, p. 97-104.

[2] Fratini L., Lo Casto S., Lo Valvo E., A technical note on an experimental device to measure friction coefficient in sheet metal forming, J. Mat. Proc. Technol., vol. 172, no. 1, 2006, p.
16-21.

[3] Lee B.H., Keum Y.T., Wagoner R.H., Modeling of the friction caused by lubrication and surface roughness in sheet metal forming, J. Mat. Proc. Technol., vol. 130-131, 2002, p. 60-63.

[4] Guo B., Gong F., Wang C., Shan D., Size effect on friction in scaled down strip drawing, J. Mater. Sci., vol. 45, no. 15, 2010, p. 4067-4072.

[5] Pacana A., Korzyński M.: Roller burnishing parameters analyse with Taguchi method. Międzynarodowa Konferencja Naukowa "MECHANIKA 2002", Rzeszów, July 2002, s. 273 279, ISSN 0209-2689

[6] Pacana A., Noga S.: Application neural networks to find the relation between the roller burnishing parameters and the surface roughness. Computer science for design and technology 2000 (Конгресса; Конструкторско технологическая информатика 2000), Моссош, СТАНКИН, 3-6.10.2000 s.292-294 ISBN 5-7028-0117-2

[7] Hertz J., Krogh A., Palmer R. G., Wstęp do teorii obliczeń neuronowych, WNT, Warszawa, 1993.

[8] Tadeusiewicz R., Sieci neuronowe, WNT, Warszawa 1998.

[9] Michalewicz Z., Algorytmy genetyczne + struktury danych = programy ewolucyjne, WNT, Warszawa, 1999.

[10] Lula P., Tadeusiewicz R., STATISTICA Neural Networks PL, Przewodnik problemowy, Statsoft, Kraków, 2001.

[11] StatSoft, Inc. Manual of STATISTICA Neural Networks Software. StatSoft Inc., Tulsa, 1998.

[12] Stachowicz F., Trzepieciński T., ANN application for determination of frictional characteristics of brass sheet metal, Journal of Artificial Intelligence, Vol. 1, No 2, 2004, pp 81-90. 